\title{
Erratum to: Einstein-scalar field equation in LTB space-time: General scheme and special solutions
}

\author{
Antonio Zecca ${ }^{1,2, a, b}$ \\ 1 Dipartimento di Fisica dell'Università degli Studi di Milano - Via Celoria, 16 - 20133 Milano, Italy \\ 2 GNFM, Gruppo Nazionale per la Fisica Matematica, Milano, Italy
}

Original article: Eur. Phys. J. Plus (2018) 133: 381, https://doi.org/10.1140/epjp/i2018-12220-x

Received: 3 October 2018

Published online: 28 May 2019

(C) Società Italiana di Fisica / Springer-Verlag GmbH Germany, part of Springer Nature, 2019

After publication of the paper, the author found an error.

Formula (38) is wrong. The correct formula is the following:

$$
\dot{\xi}=\sqrt{-\frac{c_{o}}{2} \frac{1}{\xi^{4}}+\xi_{o}},
$$

such that formula (39) must be dropped.

Consequently, the three lines between formulas (55) and (56) must be substituted by:

"From (40) there results that $\ddot{\xi}>0$ for suitable choice of the sign of $c_{o}$. Moreover (38) and (40) imply:"

Accordingly, the string of words "and a late accelerated expansion" should be dropped in the Abstract, in sect. 1 and in sect. 6 .

\footnotetext{
a e-mail: antonio.zecca@mi.infn.it

b Retired from: Dipartimento di Fisica dell'Università degli Studi di Milano.
} 